\title{
MARGINAL OVERDOMINANCE IN DROSOPHILA
}

\author{
CHRISTOPHER WILLS \\ Department of Biology, University of California, San Diego, La Jolla, California 92037
}

Manuscript received July 17, 1974

Revised copy received April 9, 1975

\begin{abstract}
A reanalysis of Drosophila viability data was undertaken to determine the role of genotype-environment interactions in the maintenance of polymorphism. Between-replicate variances of viabilities in chromosomal homozygotes and heterozygotes with the same mean fitnesses were compared, with the expectation that if the heterozygote variance were on the average greater, conditional overdominance would be prevalent; if it were less, partial dominance would be prevalent; and if it were the same, marginal overdominance of the type considered by WALLACE (1968) would be the prevalent type of variation. In fact, heterozygote variance was slightly less. The work of Dempster (1955) and of Girlespie and Langley (1974) is cited to show that this situation can still lead to balanced polymorphisms. Their general model for genetic variation in populations, consistent with the viability data, is reinforced.
\end{abstract}

$\mathrm{D}$

EMPSTER (1955) pointed out that a model such as that proposed by LEvene (1953) for the maintenance of variability in different niches fit the available data better than the overdominance model for the maintenance of most polymorphism. LevenE's model had the fitness array:

Niche

Fitness

$\begin{array}{cccl} & A A & A a & a a \\ 1 & 1 & 1 & 1-s \\ 2 & 1-s & 1 & 1 \\ 3 & 1 & 1 & 1-s \\ - & \bullet & \bullet & \bullet \\ - & \bullet & \bullet & \bullet\end{array}$

If the fitnesses are measured in a single niche two thirds of the genotypic variance should be additive when the frequencies of $A$ and $a$ are 0.5. This conformed with what had been observed by RoBinson and Coмsтоск (1955) in their studies of corn; the additive variance was too great for single-gene heterosis to be the major factor in maintaining variability in corn populations.

Dempster also pointed out that the heterozygote need not be as fit as one of the homozygotes in any niche in order for the heterozygote to be fittest over all. This observation was further quantified by GiLlespie (1973), who proved that polymorphisms can be maintained in a temporally fluctuating environment so long as the geometric mean fitness of the heterozygote is greater than that of either homozygote. Gillespie and LANGiEy (1974) went further and showed 
that even if the heterozygote were exactly intermediate in fitness in each spatial niche or over time polymorphism was still possible, since under some conditions the geometric mean fitness of the heterozygote would still be greater than those of the homozygotes.

Gillespie and LANGLey (1974) listed a number of polymorphic enzymes which had been examined in the laboratory and in which heterozygote intermediacy had been observed. In our laboratory (Wills, Phelps and Ferguson 1975 ) the fitnesses of homozygotes and heterozygotes for two polymorphic enzymes in Drosophila pseudoobscura were examined in otherwise highly inbred strains under conditions designed to maximize fitness differences. Both systems, after 38 generations of inbreeding by brother-sister mating, showed heterozygote intermediacy in fitness in most of the cases where significant fitness differences were detected. Altering the environment, however, brought about in some cases a marked change in the relative order of fitnesses with the heterozygote still intermediate.

The model presented at the beginning of this paper, in which the heterozygote is equal in fitness to one or the other homozygote in a given environment, was discussed without elaboration by WALLACE (1968), who referred to it as marginal overdominance. It seems sensible that this term can be extended to cover any variation of this model in which the average or marginal fitness of the heterozygote is greater than that of the homozygotes.

In this paper I present a reanalysis of some data on chromosome viabilities from two natural populations of $D$. pseudoobscura. Viability data were collected (WILLS 1966) from two populations of $D$. pseudoobscura. Homozygotes and heterozygotes for third chromosomes isolated from these populations were examined for egg-to-adult viability using the $B l S c / L$ marker system. All the third chromosomes used in these viability studies carried the Arrowhead gene arrangement. Eight replicates were counted for each homozygous and heterozygous chromosome combination.

\section{THE IMODEL}

The essence of the presentation is that it should be possible, by comparing the genotype-environment interactions of homozygotes and heterozygotes of equal mean fitness, to distinguish between conditional heterosis and marginal overdominance. The assumptions made are:

1) Between-replicate variation in fitness estimations over and above what can be expected by chance represents interactions between the genotype and microenvironmental variation from one replicate to another.

2) The microenvironmental variation within sets of replicates may vary somewhat from one set of replicates to another, but should not vary in any systematic way. For example, the microenvironmental variation should not be consistently larger or smaller in sets of replicates of heterozygous flies than in sets of replicates of homozygous flies.

3) Because we are dealing here with blocks of genes locked into an inversion and not with single genes, it seems certain that in different microenvironments different genes within the inversion will be important in determining fitness. 
The enormous lability of selection coefficients associated with inversions when the environment is altered slightly has been amply demonstrated (WRIGHT and Dobzhansky 1946; Da Cunha 1961; Dobzhansky et al. 1955); presumably such a variety of genes is present within an inversion that virtually any microenvironmental change will bring about an alteration in selection coefficients associated with it.

4) In the array of replicates examined in the laboratory it is unlikely that more than a small number of alleles will be important in determining the variation in fitness. However, these and other alleles locked into the inversion will undoubtedly be selected in many different ways in the great variety of environments to which the flies are subjected in nature.

5) There will be some microenvironments in which the fitness of flies heterozygous for two different chromosomes will be determined by genes that happen to be the same on the two chromosomes.

Given these assumptions, we can list hypothetical arrays of selection coefficients over a range of microenvironments for three different models: conditional heterosis, marginal overdominance (in the WALLACE (1968) sense) and partial dominance. The marginal overdominance and partial dominance models may both lead to the maintenance of polymorphism provided that the conditions of Gillespie and LANGLey (1974) are met. Because different loci are important for fitness in different microenvironments, the selection coefficients are assumed to vary in magnitude from one microenvironment to another.

Conditional heterosis model:

Microenvironment

$$
\begin{array}{cc} 
& \text { Chromosomal genotype } \\
A_{1} A_{1} & A_{1} A_{2}
\end{array}
$$

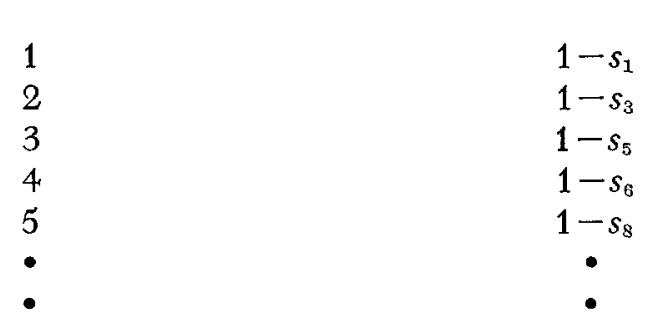

Marginal overdominance model:

Microenvironment

$$
A_{1} A_{1} \begin{array}{cc}
\text { Chromosomal genotype } \\
A_{1} A_{2}
\end{array} A_{2} A_{2}
$$

$$
\begin{aligned}
& 1 \\
& 2 \\
& 3 \\
& 4 \\
& 5
\end{aligned}
$$$$
\begin{gathered}
1 \\
1-s_{2} \\
1-s_{3} \\
1 \\
1 \\
\bullet \\
\bullet
\end{gathered}
$$

2
3
4
5

Chromosomal fitness

Chromosomal fitness

1
1
$1-s_{5}$
1
$1-s_{8}$
-

$$
1-s_{2}
$$$$
1-s_{4}
$$$$
1-s_{5}
$$$$
1-s_{7}
$$$$
1-s_{8}
$$$$
\text { - }
$$

$1-s_{1}$

1

$1-s_{3}$

$1-s_{4}$ 
Partial dominance model:

$A_{1} A_{1} \begin{array}{cc}\text { Chromosomal genotype } \\ A_{1} A_{2} & A_{2} A_{2}\end{array}$

Microenvironment

\section{Chromosomal fitness}

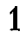

2

3

4

5

-

In each of these hypothetical arrays of selection coefficients, the selectively important locus or loci in microenvironments 3 and 5 are assumed to be homozygous in chromosomal heterozygotes. Such chance homozygosity contributes to the correlation between homozygote and heterozygote fitnesses.

In the effort to distinguish among these three models it might seem at first sight reasonable to compare particular combinations of chromosomal homozygotes and heterozygotes. There is no certainty, however, that the array of microenvironments in the set of heterozygote replicate crosses is the same as or similar to that in the set of homozygote crosses. Thus, a totally different array of genes within the inversion might be important in determining fitness in the homozygote and heterozygote crosses. It is possible to extract a relation between the models and the amount of microenvironmental variance, however, if one compares homozygotes and heterozygotes with the mean fitness (averaged over replicates), even though these may have come from crosses involving different chromosomes.

Consider first the conditional heterosis model. For $n$ microenvironments, the average of the selection coefficients for a homozygote will be $\bar{s}_{\text {hom }}=\Sigma s_{i} / n$ and the variance will be $\Sigma\left(s_{i}-\bar{s}_{\text {hom }}\right)^{2} /(n-1)$. For a heterozygote with the same mean fitness (but not necessarily involving the same chromosome), the fitness in most microenvironments will be approximately one. It will only be much less than one if the chromosomal heterozygote happens to be homozygous for a gene or genes important to fitness in that particular microenvironment. Assume that the fitness is less than one in some fraction $m$ of the microenvironments, when $0<m<n$. Then the mean of the heterozygote selection coefficients will be:

$$
\bar{s}_{\text {het }}=\frac{\sum_{j=1}^{m} s_{j}}{n}
$$

so that the average $s_{j}$ will be larger than the average $s_{i}$. 
The variance will be (remembering that both homozygote and heterozygote have the same mean fitness):

$$
s_{\text {het }}^{2}=\frac{\sum_{j=1}^{m}\left(s_{j}-\bar{s}_{\text {hom }}\right)^{2}+\sum_{j=m+1}^{n}\left(0-\bar{s}_{\text {hom }}\right)^{2}}{n-1} .
$$

This represents a biomodal distribution of $s_{j}$ 's, with a mean equal to that of the $s_{i}$ 's. Assuming that the $s_{i}$ 's have a unimodal or at the worst a uniform distribution, $s^{2}$ het should be greater than $s^{2}$ nom. Therefore, we would expect with the conditional heterosis model that the variance in fitness due to microenvironment would be greater for chomosomal heterozygotes than for chromosomal homozygotes with the same mean fitness.

We now turn to the marginal overdominance model. Suppose that for a homozygote with a mean $\bar{s}_{\text {hon }}$ there is a proportion of microenvironments $m$ in which the fitness is less than one. The homozygote variance can therefore be written in the same form as the heterozygote variance in the previous model (equation 1). In a heterozygote with the same mean fitness, what proportion of microenvironments could be expected to produce a fitness of less than one? In this model, we are not constrained as we were in the previous one by the necessity of having a smaller number of such microenvironments in the heterozygote case than in the homozygote one. The heterozygote may exhibit a very low fitness in a small fraction of the microenvironments, or a slightly lowered fitness in a large fraction. It seems likely that the proportion would average very close to $m$. Thus, the variance in fitness of chromosomal homozygotes and heterozygotes with the same average fitness would be comparable for the marginal overdominance model.

For the partial dominance model, the expectation is again different. If we again assume that a proportion $m$ of the microenvironments results in a selection coefficient for the homozygote greater than zero, the variance of the homozygotes can be written as in equation (1) above, substituting $s_{i}$ for $s_{j}$. For a heterozygote with the same mean fitness, there will be a proportion of microenvironments $p$ in which the selection coefficients will be as great on the average as those for the homozygotes (because the chromosomal heterozygote happens to be homozygous at loci important for fitness in these microenvironments), another proportion $q$ in which the selection coefficients will be smaller on the average because of the partial dominance term $h$, and the remainder in which the selection coefficients will be zero. When matched with a homozygote of comparable fitness, $p<m$ and $(p+q)>m$, since $\overline{h_{i} s_{i}}<\bar{s}_{i}$. Thus,

$$
s_{\text {het }}^{2}=\frac{\sum_{j=1}^{p}\left(s_{j}-\bar{s}_{\text {hom }}\right)^{2}+\sum_{j=p+1}^{p+q}\left(h_{j} s_{j}-\bar{s}_{\text {hom }}\right)^{2}+\sum_{j=p+q+1}^{n}\left(0-\bar{s}_{\text {hom }}\right)^{2}}{n-1}
$$

This may be compared with the formula for $s^{2}{ }_{\text {hom }}$, which represents a bimodal distribution of $s_{i}$ 's. We are safe in assuming that $s^{2}$ is approximately equal to $s^{2}{ }_{i}$, since the $s_{j}$ 's are simply instances of homozygosity for particular genes or 
groups of genes in the chromosomal heterozygote. Therefore, the trimodal distribution of selection coefficients represented by equation (2) may be expected to have a smaller variance than that of the bimodal distribution of equation (1) in this model. This leads to the prediction that partial dominance will lead to a smaller between-replicate variance for heterozygotes than for homozygotes with comparable fitness.

\section{DROSOPHILA DATA}

A total of 51 non-lethal homozygous crosses and 62 heterozygous crosses, each with eight replicates, could be employed in this analysis; the rest of the data were collected in a slightly different way. The data were transformed to remove any spurious correlation between means and variances, and also because viability data in which two classes of flies with unequal expectation are segregating within a bottle are not normally distributed, as pointed out by C. C. Cockerhain (T. MukaI, personal communication). The transformation chosen involved taking the $\log$ of $2 b / a$, where $b$ is the number of wild-type flies and $a$ the number of marker flies, then multiplying the $\log$ by integer 1,000. This was done for each sex within a bottle. The results were positive or negative numbers mostly falling between -300 and +100 , with zero representing a viability $100 \%$ of that of the marker flies. The data were checked to ensure that they were effectively normally distributed by comparing them to a $z$ distribution with Chi-square. None of the transformed data showed a significant deviation from normality.

Table 1 shows the results of an analysis of variance on the transformed data. The between-chromosome variance is significantly large for both homozygotes and heterozygotes, but more so for the former, as would be expected since homozygote viabilities cover a greater range. Between-sexes variances are not significant, but the interaction variance is significant in the heterozygotes. Consequently, in subsequent manipulations, sexes within bottles were treated separately. The error variance is a very large factor in both analyses, accounting for half the total variance in the homozygotes and almost $80 \%$ in the hetero-

TABLE 1

Analysis of variance of transformed data

\begin{tabular}{|c|c|c|c|c|c|}
\hline Source & DF & Ss & MS & $\mathbf{F}$ & F-PROB \\
\hline \multicolumn{6}{|l|}{ a) Homozygotes } \\
\hline Total & 815 & $15,018,859$ & & & \\
\hline Between chromosomes & 50 & $6,820,751$ & 136,415 & 12.95 & $<.0001$ \\
\hline Between sexes & 1 & 13,761 & 13,761 & 1.31 & n.s. \\
\hline Chromosomes $\times$ sexes & 50 & 665,693 & 13,313 & 1.26 & n.s. \\
\hline Error & 714 & $7,518,652$ & 10,530 & & \\
\hline \multicolumn{6}{|l|}{ b) Heterozygotes } \\
\hline Total & 991 & $5,605,075$ & & & \\
\hline Between chromosomes & 61 & 961,818 & 15,768 & 3.27 & $<.0001$ \\
\hline Between sexes & 1 & 52 & 52 & 0.01 & n.s. \\
\hline Chromosomes $X$ sexes & 61 & 451,979 & 7,409 & 1.53 & .005 \\
\hline Error & 868 & $4,191,226$ & 4,829 & & \\
\hline
\end{tabular}


zygotes. Further, the homozygote error mean square is over twice as large (2.18) as the heterozygote error mean square. With 714 degrees of freedom in the numerator and 868 in the denominator, this is a highly significant difference indeed.

We wish first to compare between-replicate variance within crosses, to determine whether the variance is greater than that due to chance. An increased between-replicate variance represents genotype-microenvironment interaction, since if all the replicates had exactly the same environment the same viability should be obtained from each. Walrace and Madden (1953) and Dobzhanskx and LEVENE (1955) recognized the importance of between-replicate variation as a measure of genotype-microenvironment interaction. Their method of analysis, however, did not permit a comparison of observed and expected (binomial) variation within an individual set of replicates.

Table 2 shows a typical set of data. In the table, viability estimates $\left(1000^{\circ} \log \right.$ $2 b / a)$ are obtained for each sex within each bottle, and between-replicate variance for each sex is calculated. The variance to be expected by chance on the assumption of the same chromosome viability in every replicate depends on two parameters: the average viability for the sex as a whole and the number of flies counted in each bottle. The lower the average viability, the smaller is $2 b / a$ and the greater the error variance to be expected. A small number of flies counted in one or more bottles greatly increases the variance also. Because of these complications, the expected variances were determined by simulation.

TABLE 2

Set of homozygote data, illustrating the method of analysis used

\begin{tabular}{|c|c|c|c|c|c|c|}
\hline \multirow[b]{2}{*}{ Replicate } & \multicolumn{4}{|c|}{ No. of fies wild type } & \multicolumn{2}{|c|}{$\begin{array}{l}\text { Transformed } \\
\text { viabilities }\end{array}$} \\
\hline & 우우 & $\hat{0} \hat{O}$ & ᄋ $q$ & के & 우우 & $\hat{o} \hat{\delta}$ \\
\hline 1 & 200 & 205 & 80 & 73 & -97 & -148 \\
\hline 2 & 158 & 149 & 56 & 42 & -150 & -249 \\
\hline 3 & 123 & 117 & 27 & 25 & -358 & -370 \\
\hline 4 & 150 & 130 & 56 & 35 & -127 & -269 \\
\hline 5 & 209 & 212 & 86 & 68 & -85 & -193 \\
\hline 6 & 264 & 244 & 82 & 57 & -207 & -331 \\
\hline 7 & 231 & 223 & 32 & 31 & -558 & -556 \\
\hline \multirow[t]{4}{*}{8} & 186 & 139 & 45 & 38 & -316 & -263 \\
\hline & \multicolumn{4}{|c|}{ Observed variances } & 26,742 & 15,832 \\
\hline & & \multicolumn{3}{|c|}{$\begin{array}{l}\text { Calculated variances } \\
\text { (multinominal basis) }\end{array}$} & 4,581 & 4,508 \\
\hline & & \multicolumn{3}{|c|}{$F_{(7,70)}$} & $5.83^{* *}$ & $3.59^{* *}$ \\
\hline
\end{tabular}

The transformation used was $1000^{\circ}(\log 2 b / a)$. Calculated variance was an average of 10 simulations.

*** Significant at the .01 level. 
The following parameters were defined:

$a_{i j k}=$ the observed number of marker flies of sex $i$ in replicate $j$ of cross $k$.

$b_{i j k}=$ the observed number of wild-type flies of sex $i$ in replicate $j$ of cross $k$.

$v_{i k}=2 \Sigma_{j} b_{i k} / \Sigma a_{i k}=$ the average viability of flies of sex $i$ of chromosome $k$.

$n_{i j k}=$ the total number of flies of sex $i$ in replicate $j$ of cross $k$.

$a_{i j k}^{\prime}=$ the expected number of marker flies of sex $i$ in replicate $j$ of cross $k$.

$b^{\prime}{ }_{i j k}=$ the expected number of wild flies of sex $i$ in replicate $j$ of cross $k$.

In order to calculate these expected numbers, we note that

$a^{\prime}{ }_{i j k}+b^{\prime}{ }_{i j k}=n_{i j k}$

$2 a^{\prime}{ }_{i j k} / b^{\prime}{ }_{i j k}=v_{i k}$

$2 b^{\prime}{ }_{i j k} / a^{\prime}{ }_{i j k}=v_{i k}$

Solving, $b_{i j k}^{\prime}=2 n_{i j k}$

$\widehat{v_{i k}}+2$.

The information input into the program consisted of all the $n_{i j k}$ 's for all the groups of data, along with the $v_{i k}$ 's for each group. For each sex within each replicate the program calculated $\alpha^{\prime}{ }_{i j k}$ and $b^{\prime}{ }_{i j k}$. Then, $n_{i j k}$ random numbers lying between zero and one were generated, and each was multiplied by $n_{i j k}$. The random numbers lying in the region between 0 and $a_{i j k}$ were labelled simulated marker flies; those lying between $\alpha^{\prime}{ }_{i j k}$ and $n_{i j k}$ were labelled simulated wild-type flies. A viability was calculated for each replicate generated in this fashion, transformed to 1000 times its logarithm, and the variance between replicates within sexes was calculated. This procedure was repeated ten times for each set of eight $n_{i j k}$ 's. The mean of these ten variance estimates was then calculated.

The ratio of the real variance to the variance estimated in this manner is distributed as $F$ with 7 degrees of freedom for the real and 70 for the estimated variance. If the real variance does deviate significantly from chance expectation this test has a high probability of detecting it; the .05 and .01 significance levels for 7 (numerator) and 70 (denominator) degrees of freedom are 2.14 and 2.91 respectively, compared with 2.01 and 2.64 for 7 degrees of freedom in the numerator and an infinite number in the denominator.

To reiterate, this procedure gives an estimate of the variance on the assumption that there is a single underlying viability conferred by the chromosome in question.

As would be expected, the observed variance was sometimes smaller and sometimes greater than the estimated variance. For the 124 sets of heterozygote data (remember that the two sexes are being considered separately) 58 were smaller and 66 larger, not significantly different from chance expectation. The picture was different in the homozygotes, however; here out of 102 comparisons 17 were smaller and 85 larger.

A few observed values were significantly smaller than the expected. Out of 226 sets of data, two were significantly smaller at the .05 level and two at the .01 level. One would expect about 5 and 1 respectively, and this close correspondence indicates that the simulation approach has given an accurate estimate of the expected variances. 
One would also expect about 5 and 1 significantly larger at the .05 and .01 levels respectively. In fact, there were 28 and 25 , respectively, presumably mostly crosses in which genotype-microenvironment interaction was significant. Their distribution with respect to viability is informative, and is given in Table 3. In this table the homozygotes and heterozygotes were ordered from lowest to highest viability and then broken up into groups of 20 (the last group of homozygotes has 22 and the last group of heterozygotes 24 ). There is a slight tendency for the number and size of significant homozygote comparisons to decrease with increasing viability, while those for the heterozygotes remain approximately constant over the viability range. There may also be a tendency for the number of comparisons greater than one to drop with increasing viability in the homozygotes, though again no such trend is apparent in the heterozygotes.

Do these significant $F$ values really represent interactions of the genotype with microenvironmental variation? Presumably, if the environment differs from one replicate to the next, males and females within a replicate should tend to be affected in the same way. The greater the degree to which micorenvironmental heterogeneity interacts with the genotype, the stronger this correlation should be.

To test this, male and female viabilities were recalculated from the transformed data as deviations from the mean male and female viabilities of each set of eight replicates. Correlations were calculated "within sets", thus losing an additional degree of freedom for each set of replicates. The correlation coefficients obtained for the data having significant and non-significant $F$ values are pre-

TABLE 3

Comparisons of observed and expected between-replicate variances

\begin{tabular}{llcc}
\hline $\mathrm{n}$ & $\begin{array}{c}\text { Mean } \\
\text { viability }\end{array}$ & $\begin{array}{c}\text { No of signif. } F \text { values } \\
.05 \text { level }\end{array}$ & No. of $F$ values \\
\hline
\end{tabular}

a) Homozygotes and heterozygotes were arranged in order of increasing viability, then broken up into groups of approximately 20 .

$\begin{array}{cll}\begin{array}{c}\text { Homozygotes } \\ \text { Group } 1\end{array} & 20 & 0.592 \pm .087 \\ 2 & 20 & 0.755 \pm .026 \\ 3 & 20 & 0.825 \pm .023 \\ 4 & 20 & 0.895 \pm .019 \\ 5 & 22 & 1.02 \pm .074\end{array}$

Heterozygotes

$\begin{array}{rll}\text { Group } 1 & 20 & 0.839 \pm .037 \\ 2 & 20 & 0.905 \pm .014 \\ 3 & 20 & 0.94 \pm .0099 \\ 4 & 20 & 0.972 \pm .012 \\ 5 & 20 & 1.011 \pm .011 \\ 6 & 24 & 1.077 \pm .040\end{array}$

$\begin{array}{llll}1 & 8 & 0 & 20 \\ 5 & 3 & 5 & 15 \\ 2 & 3 & 3 & 17 \\ 4 & 3 & 3 & 17 \\ 2 & 2 & 6 & 16\end{array}$

\section{0}

15

b) Comparison between matched set of homozygotes and heterozygotes (see text).

\begin{tabular}{lrrrrrr} 
Homozygotes & 47 & $.935 \pm .092$ & 8 & 3 & 9 & 38 \\
Heterozygotes & 47 & $.934 \pm .089$ & 4 & 2 & 22 & 25 \\
\hline
\end{tabular}


sented in Table 4. The largest correlation was found between homozygote males and females in which both the $F$ values were significant. Microenvironmental heterogeneity affected flies carrying these chromosomes very strongly.

The next step in the analysis was to compare homozygotes with heterozygotes of similar fitness, to determine which of the three models discussed earlier most closely fit the data. Because the homozygotes and heterozygotes had different distributions of fitness, the two sets of data are not directly comparable. The ranked fitnesses of homozygotes and heterozygotes were compared, and 47 pairs were picked in which the fitnesses were matched to one part in 1,000. The distribution of $F$ values for this matched set of homozygotes and heterozygotes is given in the second part of Table 3. Although almost twice as many $F$ values were significantly greater than 1 in the homozygote than the heterozygote data, this difference is itself not significant. There is, however, a significant excess of $F$ values greater than 1 in the homozygote data (though fewer than a third of these are individually significant). In the heterozygote data there is not. The majority of individually significant $F$ values are found among the least fit homozygotes, for which there are no matching heterozygotes and which were therefore not included in this comparison. This observation will be returned to in the Drscussion.

When analyses of variance were performed on these matched sets of data, the homozygote error variance was significantly greater than that for the heterozygotes $\left(F_{329,329}=1.26\right)$. Some of this difference can be accounted for by the fact that the homozygotes produced fewer flies per bottle than did the heterozygotes, thus increasing the apparent error variance. This in turn is probably due to the fact that the parents of the homozygote crosses were more inbred than those of the heterozygote crosses. The $F$-ratio analysis given above, which factored out differences due to size of cultures, also showed slightly but significantly more between-replicate variance among the homozygotes and thus favors the partial dominance model. The differences between the matched homozygote and heterozygote data, however, are slight.

\section{TABLE 4}

"Within sets of replicates" correlations between viabilities of males and females in the same bottle

Homozygotes

Significant between-replicate variance in:

$\begin{array}{lrr}\text { a) Neither sex } & 173 & +.296^{* *} \\ \text { b) One sex } & 124 & +.286^{* *} \\ \text { c) Both sexes } & 54 & +.807^{* *}\end{array}$

Heterozygotes

Significant between-replicate variance in:
a) Neither sex
292
$+.106$
b) one or (one case only) both sexes
138
$+.252^{* *}$

The stronger the correlation, the greater the presumed role of microenvironmental variation.

** Significant at the .01 level. 
DISCUSSION

The analysis given above depends on the assumptions listed at the beginning of the paper. The assumption that different genes within the inversion are selectively important in different microenvironments is a crucial one, and leads to a consistent interpretation of viability data from natural populations.

If the partial dominance model is the correct one, one might expect a strong correlation between homozygote and heterozygote viabilities. Two contrasting sets of results from the same organism, $D$. pseudoobscura, illustrate why this is not necessarily expected. DoвzHansky and Spassky (1963) found no correlation between homozygote and heterozygote viabilities, counting one or two replicates per chromosome. WILLS (1966) found a significant correlation, counting eight replicates per chromosome with the same total number of flies. If mocroenvironmental variation occurs among replicates within a cross, it is certain also to be present among the different bottles in which homozygote and heterozygote viabilities are being measured. With a small number of replicates, it is unlikely that the same array of genes is important in viability in both the homozygote and heterozygote crosses; the probability of some overlap increases as the number of replicates goes up.

The microenvironmental variation between replicates gives us a hint of the effect larger environmental variations would have on viability estimates. It seems certain that had the environment in the experiment cited here been radically changed - by altering the yeast or the temperature, for example-the order of fitnesses of the homozygote and heterozygote chromosomes would also have altered completely. It is striking that the "unfit" homozygotes in these data all showed strong genotype-microenvironment interactions; slight changes in environment were enough to shift their fitnesses markedly. Eight out of 20 of the homozygotes with the lowest fitnesses had $F$ values significant at the .01 level. If genes with a harmful effect on fitness are to be preserved in a natural population, it is to be expected that those whose deleterious effects are confined to a small range of environments will more likely be retained. They are less likely to be removed by selection than those whose deleterious effects extend over a wide range of environments.

This leads to the prediction that there should be small between-replicate error variances associated with newly-induced variation, as contrasted with variation retained in natural populations (see Blatherwick and Wills 1971). The magnitude of the between-replicate variances of EMS-induced variation examined by MUKaI (1970) or the X-ray induced variation examined by WALLACE (1963) may be instructive on this point. Between-replicate variance may also be a useful measure to determine whether natural selection has acted on the spontaneous variation accumulated over balancer chromosomes by MuKaI (1964); large between-replicate variances may indicate that it has.

It has not been possible to distinguish unequivocally between two of the three models of genetic variation set forth at the outset. The possibility cannot be ruled out that a mixture of conditionally heterotic and highly dominant genes could have led to the results reported here. It seems likely, however, that wide- 
spread conditional heterosis can be discounted, at least under these laboratory conditions. Marginal overdominance in the WALLACE (1968) sense also seems unlikely, and a slight partial dominance seems to predominate. As noted by Dempster (1955) and worked out in detail by GILLESPIE and LANGLey (1974), however, even partial dominance may lead to the maintenance of polymorphisms if genotype-environment interaction is high. Published measures of partial dominance in natural populations range from 0.177 to 0.489 (MUKAI and YamAGUCHr 1974). Most are less than the 0.5 of no dominance, and would therefore easily lead to marginal overdominance in the broader sense. Indeed, the degree of partial dominance is itself dependent on the severity of the environment (WILLS 1966).

In sum, a fuller understanding of genetic variation in natural populations can be gained by considering the effects of fluctuating environments. Evidence for slight partial dominance, which might without this consideration lead to a "classical" view of population structure, can be seen to be consistent with a "balanced" view.

I thank Drs. Everetr Dempster, Michael Gilpin, Terumi Mukai and Michael Soulé for valuable discussions. Some of the work cited in this paper was supported by the Public Health Service.

\section{LITERATURE CITED}

BLATHERWick, C. and C. WILLs, 1971 The accumulation of genetic variability in yeast. Genetics 68: 547-557.

DA Cunha, A. B., 1951 Modification of the adaptive values of chromosomal types in Drosophila pseudoobscura by nutritional variables. Evolution 5: $395-404$.

Dempster, E. R., 1955 Maintenance of genetic heterogeneity. Cold Spring Harbor Symp. Quant. Biol. 20: 25-32.

Dobzhansky, Th. and H. Levene, 1955 Genetics of natural populations. XXIV. Developmental homeostasis in natural populations of Drosophila pseudoobscura. Genetics 40: 797-808.

Dobzransky, Trr. and B. Spassky, 1963 Genetics of natural populations. XXXIV. Adaptive norm, genetic load and genetic elite in Drosophila pseudoobscura. Genetics 48: 1467-1486.

Dobzhanski, Тh., O. Pavlovsky, B. Spassky and N. Spassky, 1955 Genetics of natural populations. XXIII. Biological role of deleterious recessives in populations of Drosophila pseudoobscura. Genetics 40: 781-796.

Gillespie, J. H., 1973 Polymorphism in random environments. Theoret. Pop. Biol. 4: 193-195.

Gillespie, J. H. and C. H. LANGLey, 1974 A general model to account for enzyme variation in natural populations. Genetics 76: $837-848$.

Levene, H. 1953 Genetic equilibrium when more than one ecological niche is available. Am. Naturalist 87: 331-333.

Mukax, T,, 1964 The genetic structure of natural populations of Drosophila melanogaster. I. Spontanenous mutation rate of polygenes controlling viability. Genetics 50: 1-19. -_-, 1970 Viability mutations induced by ethyl methanesulfonate in Drosophila melanogaster. Genetics 65: 335-348.

Mukai, T. and O. YamaGUChi, 1974 The genetic structure of natural populations of Drosophila melanogaster. XI. Genetic variability in a local population. Genetics 76: 339-366.

Robrnson, H. F. and R. E. Сомsтock, 1955 Analysis of genetic variability in corn with reference to probable effects of selection. Cold Spring Harbor Symp. Quant. Biol 20: $127-$ 136. 
Wallace, B., 1968 pp. 213-214. In: Topics in Population Genetics. Norton, New York.

1963 Further data on the over-dominance of induced mutations. Genetics 48: 633-651.

Wallace, B. and C. Madden, 1953 The frequencies of sub- and supervitals in experimental populations of Drosophila melanogaster. Genetics 35: 456-470.

Wilus, C., 1966 The mutational load in two natural populations of Drosophila pseudoobscura. Genetics 53 : 281-294.

Wrlus, C., J. Phelps and R. Ferguson, 1975 Further evidence for selective differences between isoalleles in Drosophila. Genetics 79: 127-141.

Wright, S. and Th. Dobzhansky, 1946 Genetics of natural populations. XII. Experimental reproduction of some of the changes caused by natural selection in certain populations of Drosophila pseudoobscura. Genetics 31 : 125-156.

Corresponding editor: R. W. ALLARD 